\title{
Functional recovery and depression mitigation in a case study of chronic stroke receiving intensive, long-duration mobility/fitness training
}

\author{
Catherine Boissoneault ${ }^{1}$, Anna Khanna ${ }^{1}$ and Janis J Daly ${ }^{1,2 *}$ \\ ${ }^{1}$ Department of Neurology, College of Medicine, University of Florida, Gainesville Florida, Florida \\ ${ }^{2}$ Brain Rehabilitation Research Center, North Florida/South Georgia VA Medical Center, Gainesville Florida, Florida
}

\begin{abstract}
Purpose: Depression is prevalent among stroke survivors and is associated with a number of adverse health outcomes. However, for those with severe depression after chronic stroke, there is little to no information regarding response of depression to exercise addressing persistent physical impairments and dysfunction or response to exercise therapy for those who are severely depressed.

Methods: We enrolled a male stroke survivor at 1.75 years post stroke, with severe depression. Measures collected at pre- and post-treatment were as follows: Beck Depression (mood); Fugl-Meyer (FM joint movement coordination); Berg Balance Scale (BBS); Timed Up and Go (TUG; mobility); Functional Independence Measure (FIM; subscales of Self-Care, Transfer, Locomotion); Craig Handicap Assessment and Reporting Technique (CHART; quality of life assessment of how patients with disabilities function in the community and at home); 36-Item Short Form Survey (SF36; quality of life measure); and the Stroke Impact Scale (SIS stroke-specific quality of life measure). Exercise was scheduled as follows: 6 mo therapy, five times/wk (1-2.5hrs/session); and additional $6 \mathrm{mo}$, 2-3times/wk, and included aerobic exercise and coordination/strength training for balance and gait. The treatment protocol included exercises for balance and coordination of the lower limb, strengthening, gait coordination, and aerobics exercise on a stationary cycle.

Results: Depression improved from severe to mild. The initial presence of severe depression did not preclude significant improvement in mobility and life role participation. Clinically significant gains were exhibited, not only in depression, but also in balance, mobility, and activities of daily living. Moreover, his quantified changes included clinically significant progression to categories of 'low fall risk' and 'functionally independent'. He achieved important clinical and functional goals, as reflected by improved scores in measures of life role participation and personal milestones.
\end{abstract}

Conclusion: Though this is a case study, it is reasonable to consider that a long-duration exercise program can be of great benefit in terms of functional and quality of life gains, regardless of the initial presence of severe depression.

\section{Introduction}

Depression is prevalent among stroke survivors and is associated with a number of adverse health outcomes, including: poor quality of life, limited participation in social activities, limitation in activities of daily living (ADLs), and notably, reduction in rehab treatment efficacy [1]. In the non-stroke population, depression responds to exercise and is associated with balance impairment and level of physical activity [2]. In a review of studies on stroke survivors with depression, there was only a small beneficial effect on depression after 4-12wks of exercise [3]. One study of stroke survivors $(n=3)$ with only mild depression found some improvement in depression after repetitive transcranial magnetic stimulation and aerobics [4]. But for those with severe depression in chronic stroke, there is little to no information regarding response of depression to longer-duration exercise addressing persistent physical impairments and dysfunction. Given the dearth of information, we quantified baseline and response to treatment for a case of severe depression in a chronic stroke survivor who received long-duration exercise and mobility training.

\section{Methods}

\section{Participant}

We enrolled a male stroke survivor, age 64years, with history of depression. The study was conducted according to the Declaration of Helsinki and with oversight of the University of Florida Internal Review Board, including written informed consent of the subject. At entry into the study, he was 1.75 years post-embolic stroke involving left basal ganglia, left frontal, parietal, occipital cortices, and right inferior frontal

${ }^{\star}$ Correspondence to: Daly JJ, Ph.D., Professor, Department of Neurology, College of Medicine, University of Florida, Director, Brain Rehabilitation Research Center, North Florida/South Georgia VA Medical Center, Florida, Tel: 1-216-402-8940; E-mail: janis.daly@neurology.ufl.edu

Key words: stroke, depression, post stroke depression, quality of life, mobility, function

Received: March 03, 2019; Accepted: March 11, 2019; Published: March 14, 2019 
cortex. Co-morbidities included: severe depression (Beck Depression Index $(\mathrm{BDI})=31)$, HIV, hepatitis C, Type 2 diabetes, hypertension, and hyperlipidemia (Medications, Table 1). History included one-week acute care after the stroke, three weeks inpatient rehab, home health, and outpatient physical therapy. At study enrollment, his medications included long-standing citalopram for depression at $20 \mathrm{mg} /$ day (Table 1).

\section{Treatment}

The intervention schedule was as follows: 6mo therapy, five times/ wk (1-2.5hrs/session); and additional 6mo, 2-3times $/$ wk. Moderate aerobic exercise was conducted on a stationary cycle (twice/wk), using standard methods, including safety criteria of resting blood pressure $\leq 140 / 90$. Strength, balance, and coordination exercises were assigned according to established, conventional guidelines and surface functional electrical stimulation (FES) was used according to standard clinical practice to guide re-training of voluntary muscle activation (sidelying, sitting, and standing). Strengthening and gait coordination training was administered according to our protocol which, in prior work, produced gait coordination improvement in chronic stroke $[5,6]$.

\section{Measures}

The Beck Depression Index (BDI) assessed depression. We measured the following variables: static and dynamic balance (Berg Balance Scale (BBS); joint movement coordination (Fugl-Meyer (FM)); static and dynamic balance (Berg Balance Scale (BBS); functional independence $>45 / 56$ ) [7] functional mobility (Timed Up and Go Test (TUG); fall risk, $>14 \mathrm{sec}$ ) [8], patient disability (Functional Independence Measure (FIM) [9] subscales of Self-Care, Transfers, and Locomotion); quality of life and life role participation (Craig Handicap Assessment Rating Tool (CHART) [10] subscales of physical and cognitive independence and occupation). We used two mixed measures (impairment, function, life role): SF 36 subscales [11] of emotional well-being, social function, general energy, and health; and Stroke Impact Scale (SIS) [12] subscales of memory, ADL/instrumental activities of daily living (IADL), mobility, life role participation, and subjective stroke recovery.

\section{Baseline}

This participant presented with severe depression (BDI, 31) [13], though he had a long-standing prescription for the anti-depressant, citalopram (20mg/day). He had weakness of ankle and hip muscles; lower limb dyscoordination (FM, 15/34); gait speed indicating only 'household ambulator' $(.38 \mathrm{~m} / \mathrm{sec})$; balance and mobility scores indicative of high fall risk (BBS, 35/56 and TUG, 27.9secs); physical de-conditioning; restricted activities of daily living; and isolated social realm

\section{Improved impairments, function, and quality of life}

Depression improved from severe at baseline (BDI, 31) to mild at post-treatment (BDI, 14; Table 2), and impairment improved in limb movement coordination, balance, functional mobility (Table 2) and exercise endurance. Cardiovascular function was too poor initially for participation in cycle exercise (his resting blood pressure was $>140 / 90$, ranging (136-156)/(92-102)), but improved sufficiently by week three to at least begin to participate. His cycling tolerance was limited to 5 minutes for the first 10 days (Rate of Perceived Exertion (RPE), 8/10); however he gradually increased exercise endurance, in 5-10-minute increments over $5 \mathrm{mos}$, finally achieving $45 \mathrm{mins}$ of exercise endurance at moderate perceived effort (4-6/10 RPE). He began cycling twice weekly at his community gym, with independent monitoring of his
Table 1. Medications

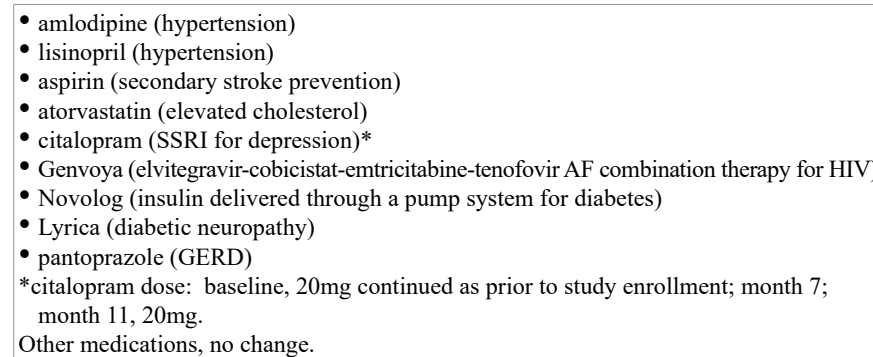

Other medications, no change.

Table 2. Balance, Mobility, Coordination, and Mood Improvement

\begin{tabular}{|l|c|c|c|c|}
\hline & Pre & Post & $\begin{array}{c}\text { Change } \\
\text { Score } \\
\text { (Post-Pre) }\end{array}$ & MDC \\
\hline BBS (55 points, normal) & 35 & 52 & 17 & $4.13^{14}$ \\
\hline TUG (secs) & 27.95 & 16.88 & -11.07 & $3.16^{15}$ \\
\hline FM-LE (34 points, normal) & 15 & 23 & 8 & $3.57^{15}$ \\
\hline Beck Depression Index (points) & 31 & 14 & -17 & \\
\hline
\end{tabular}

$\mathrm{MDC}=$ Minimum detectible change, indicating a quantitatively measurable change.

heart rate. Function improved according to SIS subdomains of ADL/ IADL and Mobility (Table 3a); SF36 Energy/Fatigue subscale (Table 3b), and FIM subdomains of Self-Care, Transfers, and Locomotion (Table 4a). Personal milestones, 'firsts' since his stroke (Table 4b), included improved walking confidence, IADL's, transferring to and from floor to play with his dog, and greater walking endurance. Quality of life improved according to the CHART domains of Occupation, Cognitive Independence, Physical Independence, and total CHART score (Table 4c); and SIS domain of Participation/Role Function (Table 3a). Improvements on these measures were reflected in personal milestones such as return to driving, arranging for and participation in group exercise class, stroke support group, attendance at local gym for cycling and exercises, attendance at YMCA for swimming independently. $\mathrm{He}$ went on a beach vacation, which was reportedly very important to him (Table 4b). Depression improved from severe at baseline (BDI, 31) to mild at post treatment 12 (BDI, 14; Table 2). Observable and reported improvements in mood occurred gradually, with behavioral signs of lessening depression observable only beginning at month 6 of the study and continuing to occur concurrently with ongoing achievement of personal life role milestones through month 12. Antidepressant was managed through his primary care facility, as follows: at month 1 of the study, 20mg citalopram; months 7-10, 40mg; and months 11-12, decreased back to his usual dose of 20mg (Table 1) [14-16].

\section{Discussion}

This case contributes to the literature by quantifying improvement in mobility, function, and life role participation for a chronic stroke survivor who began the neurorehabilitation program with severe depression (BDI, $>31$ ). The program was a long-duration, comprehensive exercise and mobility training and management of antidepressant medication. During the course of the neurorehabilitation program, depression improved from severe to mild. Proposed mechanisms of effects of exercise on mood include boosted immune function or elevated cortisol levels [3,17]. Initially, this participant was extremely deconditioned, and after six months, exercise endurance increased to $45 \mathrm{~min}$. He independently monitored heart rate, and began twice weekly cycling at a community gym, became independent with strengthening exercise and aquatic exercise. In contrast to our work, other studies reported only a small effect on depression in subacute/ 
Table 3. Mixed Measures of Impairment, Function, and Quality of Life.

\begin{tabular}{|c|c|c|c|c|c|}
\hline $\begin{array}{l}\text { 3.a. Stroke Impact Scale (mixed measure of impairment, } \\
\text { function and life role participation) }\end{array}$ & Baseline & Post & $\begin{array}{l}\text { Change Score } \\
\text { (Post-Pre) }\end{array}$ & MDC $^{16}$ & MCID $^{16}$ \\
\hline SIS (total) & 384.92 & 581.33 & 196.41 & - & - \\
\hline SIS - Memory and Thinking & 42.86 & 71.43 & 28.57 & - & - \\
\hline SIS - ADL/IADL & 75 & 85 & 10 & 17.3 & 5.9 \\
\hline SIS - Mobility & 83.33 & 94.44 & 11.11 & 15.1 & 4.5 \\
\hline SIS - Participation/Role Function & 21.88 & 87.5 & 65.62 & - & - \\
\hline SIS - Stroke Recovery & 30 & 75 & 45 & - & - \\
\hline \multicolumn{6}{|l|}{$\mathrm{MDC}=$ minimum detectible change $\mathrm{e}^{10}$} \\
\hline \multicolumn{6}{|l|}{ MCID=minimal clinically important difference (or change) ${ }^{10}$} \\
\hline 3.b. SF-36 & Baseline* & Post & $\begin{array}{l}\text { Change Score } \\
\text { (Post-Pre) }\end{array}$ & & \\
\hline SF-36 (global average $(0=$ poor quality of life) & 24.94 & 68.46 & 43.52 & & \\
\hline SF36 - Role Limitations due to emotional problems & 0 & 66.67 & 66.67 & & \\
\hline SF36 - Energy/Fatigue & 15 & 50 & 35 & & \\
\hline SF36 - Emotional Well-being & 32 & 96 & 64 & & \\
\hline SF36 - Social Functioning & 25 & 87.5 & 62.5 & & \\
\hline SF36 - Pain & 32.5 & 77.5 & 45 & & \\
\hline SF36 - General Health & 40 & 80 & 40 & & \\
\hline
\end{tabular}

Table 4. Measures of Function, Personal Milestones, and Quality of Life.

\begin{tabular}{|c|c|c|c|}
\hline \multicolumn{4}{|c|}{ 4.a. Functional Independence Measure (FIM) } \\
\hline FIM Domains & Baseline & Post & Change Score \\
\hline Self Care & 37 & 41 & 4 \\
\hline Transfers & 18 & 20 & 2 \\
\hline Locomotion & 11 & 12 & 1 \\
\hline \multicolumn{4}{|c|}{ 4.b. Milestones Achieved in Personal Functional and Life Role Participation } \\
\hline \multicolumn{4}{|c|}{ - Improved confidence with walking } \\
\hline \multicolumn{4}{|c|}{ - Independence with IADLs (laundry, meal prep, housekeeping) } \\
\hline \multicolumn{4}{|c|}{ - Independent with transferring to/from floor to play with dog } \\
\hline \multicolumn{4}{|c|}{ - Eliminated soda from diet and incorporated more water } \\
\hline \multicolumn{4}{|c|}{ - Improved aerobic capacity (tolerates 45 min cycling at moderate intensity) } \\
\hline \multicolumn{4}{|c|}{ - Walked $\sim 1 / 4$ mile to neighbor's home } \\
\hline \multicolumn{4}{|l|}{ - Returned to driving } \\
\hline \multicolumn{4}{|c|}{ - Regular participation in stroke support group ( $2 \mathrm{x} / \mathrm{month})$} \\
\hline \multicolumn{4}{|c|}{ - Regular attendance at local YMCA to independently cycle, perform strengthening exercises (home exercise program (HEP)), swim, and attend group classes } \\
\hline \multicolumn{4}{|c|}{ - Beach vacation in St. Augustine with overnight stay at hotel ( $1^{\text {st }}$ vacation and trip to beach since stroke) } \\
\hline \multicolumn{4}{|c|}{ - Regular use of memory aides to facilitate compliance with HEP (alarms, voice-reminders on phone, written reminders in notebook) } \\
\hline $\begin{array}{l}\text { 4.c. Craig Handicap Assessment Rating } \\
\text { Tool (CHART) }\end{array}$ & Baseline & Post & Change Score (Post-Pre) \\
\hline CHART total (total possible, 600 ) & 406 & 522.7 & 116.7 \\
\hline CHART - Physical Independence & 75 & 99.2 & 24.2 \\
\hline CHART - Cognitive Independence & 34 & 80 & 46 \\
\hline CHART - Occupation & 12 & 43.5 & 31.5 \\
\hline
\end{tabular}

acute stroke survivors after exercise [3]. To our knowledge, there are no available studies of severe depression in chronic stroke and mitigation of depression from severe to mild, in response to a neurorehabilitation program.

This participant also improved in coordination, balance, functional mobility, and life role participation. It has long been established that there are complex inter-relationships among those domains. Indeed, others have shown that all three domains of impairment, function, and life role participation are critically related for chronic stroke survivors ( $>6 \mathrm{mos}$ ). For example, Carod-Artal and team [18] reported correlations not only between impairment and function measures, but also between function and life role participation measures.

Given the complex interactions within and among impairment, function, and life role participation measures, it appears imperative to provide treatment that comprehensively addresses the array of existing problems for any patient. This may explain the demonstrated gains for this participant. As he progressed in balance, coordination, mobility, and endurance, he consequently improved independence in both ADL and IADL. BBS score improved by 17 points, far exceeding the minimal detectible change (MDC), and also elevated him to the categories of "functional independence" ( $>45 / 56)$ and "low fall risk" (>40/56; Table 2). With the demonstrated gains in impairment, his function improved. His SIS ADL/IADL improved at almost double the minimum clinically important difference (MCID), and his SIS Mobility score improved at almost triple the MCID (Table 3a). Similarly, gains in coordination, balance, and mobility likely imparted greater confidence to participate in social activities within the community (e.g. group exercise class, stroke support group, visiting friends and neighbors; Table 4b)). All of these physical function and quality of life improvements likely contributed to his improved depression. 


\section{Conclusion}

Though this is a case study, it is reasonable to consider that a longduration exercise program can be of great benefit in terms of functional and quality of life gains, regardless of the initial presence of severe depression.

\section{Funding Acknowledgement}

This study received no specific grant from any funding agency in the public, commercial, or not-for-profit sectors; rather, it was funded by the following: McKnight Brain Institute, College of Medicine, College of Public Health and Health Professions, University of Florida, Gainesville Florida.

\section{Declaration of conflict of interest}

The authors have no conflicts to declare

\section{References}

1. Gillen R, Eberhardt TL, Tennen H (1999) Screening for depression in stroke: relationship to rehabilitation efficiency. J Stroke Cerebrovasc Dis 8: 300-306.

2. Thilarajah S, Mentiplay BF, Bower KJ (2017) Factors associated with post-stroke physical activity: a systematic review and meta-analysis. Arch Phys Med Rehabil 1-14

3. Eng JJ, Reime B (2014) Exercise for depressive symptoms in stroke patients: a systematic review and meta-analysis. Clin Rehabil 28: 731-739. [Crossref]

4. VanDerwerker CJ, Ross RE, Stimpson KH (2018) Combining therapeutic approaches: rTMS and aerobic exercise in post-stroke depression: a case series. Top Stroke Rehabil 25: 61-67.

5. Daly JJ, McCabe JP, Gansen J, Rogers J, Butler K, et al. (2012) Gait Coordination Protocol for recovery of coordinated gait, function, and quality of life following stroke. J Rehabil Res Dev 49: 11-28. [Crossref]

6. Daly JJ, Zimbelman J, Roenigk KL (2011) Recovery of coordinated gait: randomized controlled stroke trial of functional electrical stimulation (FES) versus no FES, with weight-supported treadmill and over-ground training. Neurorehabil Neural Repair 25 : 588-596.
7. Shumway-Cook A, Baldwin M, Polissar NL, Gruber W (1997) Predicting the probability for falls in community-dwelling older adults. Phys Ther 77: 812-819. [Crossref]

8. Andersson AG, Kamwendo K, Seiger A, Appelros P (2006) How to identify potentia fallers in a stroke unit: validity indexes of 4 test methods. J Rehabil Med 38: 186-191. [Crossref]

9. Granger CV, Cotter AC, Hamilton BB, Fiedler RC (1993) Functional assessment scales: a study of persons after stroke. Arch Phys Med Rehabil 74: 133-138. [Crossref]

10. Whiteneck GG, Charlifue SW, Gerhart KA, Overholser JD, Richardson GN (1992) Quantifying handicap: a new measure of long-term rehabilitation outcomes. Arch Phys Med Rehabil 73: 519-526. [Crossref]

11. McHorney CA, Ware JE Jr, Raczek AE (1993) The MOS 36-Item Short-Form Health Survey (SF-36): II. Psychometric and clinical tests of validity in measuring physical and mental health constructs. Med Care 31: 247-263. [Crossref]

12. Duncan PW, Wallace D, Lai SM, Johnson D, Embretson S, et al. (1999) The stroke impact scale version 2.0. Evaluation of reliability, validity, and sensitivity to change. Stroke 30: 2131-2140.

13. Kotila M, Numminen H, Waltimo O, Kaste M (1998) Depression after stroke: results of the FINNSTROKE Study. Stroke 29: 368-372. [Crossref]

14. Flansbjer UB, Blom J, Brogårdh C (2012) The reproducibility of Berg Balance Scale and the Single-leg Stance in chronic stroke and the relationship between the two tests. PM R 4: 165-170. [Crossref]

15. Hiengkaew V, Jitaree K, Chaiyawat P (2012) Minimal detectable changes of the Berg Balance Scale, Fugl-Meyer Assessment Scale, Timed "Up \& Go" Test, gait speeds, and 2-minute walk test in individuals with chronic stroke with different degrees of ankle plantarflexor tone. Arch Phys Med Rehabil 93: 1201-1208.

16. Lin KC, Fu T, Wu CY (2010) Minimal detectable change and clinically important difference of the Stroke Impact Scale in stroke patients. Neurorehabil Neural Repair 24: 486-492.

17. Winstein CJ, Stein J, Arena R (2016) Guidelines for adult stroke rehabilitation and recovery: a guideline for healthcare professionals from the American Heart Association/ American Stroke Association. Stroke 47: e1-e72.

18. Carod-Artal FJ, Egido JA (2009) Quality of life after stroke: the importance of a good recovery. Cerebrovasc Dis 27: 204-214. [Crossref]

Copyright: $\bigcirc 2019$ Boissoneault C. This is an open-access article distributed under the terms of the Creative Commons Attribution License, which permits unrestricted use, distribution, and reproduction in any medium, provided the original author and source are credited. 